\title{
INVESTIGACIONES
}

\section{Experiencias de Evaluación en Educación Física. Una Aproximación desde la Formación Inicial del Profesorado}

\author{
Assessment Experiences in Physical Education: \\ An Approach from Teacher Education
}

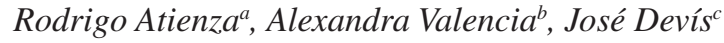 \\ a Universidad de Valencia \\ Correo electrónico: rodrigo.atienza@uv.es \\ ${ }^{\mathrm{b}}$ Universidad de Valencia \\ Correo electrónico: alexandra.valencia@uv.es \\ ${ }^{c}$ Universidad de Valencia \\ Correo electrónico: jose.devis@uv.es
}

\begin{abstract}
RESUMEN
El objetivo de este estudio es conocer las experiencias vividas por un grupo de estudiantes universitarios sobre la evaluación que se realizaba durante su periodo de educación física escolar. Para ello, se realizó un estudio cualitativo en el que participaron 45 estudiantes universitarios de la Facultad de Magisterio. Cada participante redactó un relato retrospectivo de sus experiencias y participó en dos grupos de discusión. Los resultados están organizados a partir de las tres categorías derivadas del análisis: los contenidos y conocimientos evaluados, los medios de evaluación utilizados y los criterios de evaluación aplicados. Los resultados destacan la tendencia a centrar la evaluación en aquellos contenidos hegemónicos de la asignatura (los juegos en la etapa de primaria y la enseñanza deportiva y el acondicionamiento físico en secundaria), a evaluar aisladamente los conocimientos prácticos, teóricos y actitudinales, empleando medios diferenciados para ello. También se observa que los criterios de evaluación aplicados por el profesorado están en sintonía con los propuestos por los currículos oficiales. Igualmente, se hallaron diferencias entre los modelos de evaluación empleados en cada una de las etapas educativas.
\end{abstract}

Palabras claves: evaluación, educación física escolar, educación primaria, educación secundaria.

\begin{abstract}
The aim of this study is to understand the experiences of a group of university students with the assessment carried out during their time as physical education (PE) students in grade school. A qualitative study was carried out with 45 students from the Education Faculty. Each participant wrote a retrospective account of their experiences and participated in two focus groups. The results are organized into three categories derived from the analysis: the content and knowledge assessed, the means used, and the criteria applied. The results highlight the tendency to assess the dominant contents of the subject (games in primary school and sports and fitness in secondary school) and to assess theoretical, practical, and attitudinal knowledge in an isolated manner, employing different means for each. It was also observed that the assessment criteria applied by teachers were in line with those proposed by the official curriculum. Likewise, differences were found between the assessment models used in each of the educational stages.
\end{abstract}

Keywords: assessment, physical education, primary school, secondary school. 
Estudios Pedagógicos XLIV, $\mathrm{N}^{\circ}$ 2: 127-147, 2018

EXPERIENCIAS DE EVALUACIÓN EN EDUCACIÓN FÍSICA. UNA APROXIMACIÓN DESDE LA FORMACIÓN INICIAL DEL PROFESORADO

\section{MARCO TEÓRICO}

En la formación inicial del profesorado de educación física (FIPEF) nos encontramos con un colectivo de estudiantes con experiencias adquiridas durante su escolarización que influyen sustancialmente en cómo entienden y afrontan su proceso para convertirse en docentes, es decir, las experiencias vividas y las creencias construidas durante sus años previos en el contexto de la educación formal afectan la manera que tienen de abordar su periodo universitario de formación y, también, su futuro como docentes (Gómez \& Guerra, 2012; Jhonson, 2010; López-Pastor et al., 2004). Para algunos autores, estas experiencias y creencias se convierten en conocimientos consolidados o filtros interpretativos que condicionan su desarrollo como estudiantes y futuros profesores o profesoras (Doolittle, Dodds \& Placek, 1993; Martínez, 1994). Dichas experiencias pueden resultar tan relevantes en la formación inicial como la adquisición de conocimientos profesionales, pues comprender cómo se han ido constituyendo es el paso previo para su deconstrucción y transformación (Tillema, 2000).

Uno de los aspectos educativos que se ven afectados por las experiencias y creencias de los estudiantes universitarios es la evaluación de la enseñanza y el aprendizaje en educación física (EF). El concepto de evaluación ha ido cambiando en función del contexto social, histórico, político y educativo en el que se ha desarrollado (Álvarez, 2001; López, 2014; López-Pastor, 2006). No obstante, podemos afirmar que la evaluación es un aspecto clave de todo el proceso educativo ya que acaba definiendo la enseñanza que se lleva a cabo (López-Pastor, 2006; Prieto, 2015; Santos-Guerra, 2000). De hecho, López-Pastor (2000a) entiende la evaluación como encrucijada didáctica ya que "afecta, condiciona y refleja el resto de aspectos didácticos que aparecen en cualquier proceso educativo (objetivos, contenidos, metodología, actividades, temporalización, recursos)" (p. 17). Además, la evaluación se entiende de formas distintas según la perspectiva curricular de referencia y la tradición de cada campo o área curricular concreta.

Mucho se ha investigado sobre la enseñanza de la EF, pero poco sobre la evaluación, y menos desde una aproximación cualitativa. Existen múltiples definiciones de la evaluación que han ido pasando de un posicionamiento tradicional, cuyo principal protagonista era el profesorado, hacia un enfoque actual denominado como alternativo, en el que tanto el profesorado como el alumnado son plenamente partícipes del proceso evaluativo (Prieto, 2015). Estos dos enfoques responden de forma diferente a las siguientes cuestiones básicas de la evaluación: por qué evaluar, para qué evaluar, qué evaluar, cuándo evaluar, cómo evaluar, quién evalúa, a quién evalúa, para quién evaluar y a quién beneficia la evaluación. Según diversos autores (López, 2014; López-Pastor, 1999; Prieto, 2015), en el primer enfoque el maestro o maestra es la figura principal en torno a la que gira todo el proceso evaluativo con heteroevaluaciones unidireccionales (docente a discente). En cambio, en el segundo enfoque, la figura del docente pasa a segundo plano o, mejor dicho, se equipara con la figura del alumnado, ya que éste participa activamente en el proceso de enseñanza y aprendizaje.

El enfoque tradicional de evaluación-calificación en EF se corresponde de una forma clara con discursos y enfoques de una EF orientada al rendimiento, así como con un currículum por objetivos enmarcado en una racionalidad técnica que entiende la evaluación como medición, control y/o poder (López-Pastor, 2000a). Desde este prisma, en la práctica escolar la evaluación se utiliza principalmente para calificar, aparte de controlar y de utilizar 
la calificación como poder por parte del profesorado sobre el alumnado. Por el contrario, el enfoque alternativo en la EF, más participativo, se corresponde con una racionalidad práctica y se apoya en el diálogo y la comprensión. Además, enfatiza el proceso de aprendizaje más que los resultados y utiliza para tal fin una metodología cualitativa centrada en la mejora del aprendizaje (Álvarez, 2001).

Si tenemos en cuenta la diversidad de contenidos asociados a la EF, podemos distinguir, además de los enfoques, una evaluación del conocimiento teórico, una del conocimiento práctico y otra del conocimiento actitudinal. La evaluación teórica se aborda mediante exámenes o pruebas teóricas con el objetivo de equipararse a otras asignaturas que gozaban de mayor estatus académico (López-Pastor et al., 2013). En cambio, la evaluación del conocimiento práctico es más difícil de llevar a cabo y suele realizarse mediante test de habilidades motrices y de condición física, tablas con puntuaciones y el 'juicio subjetivo' del profesorado en materias como el rendimiento en el juego. Por su parte, la evaluación de las actitudes suele realizarse a través de la observación directa (Sicilia et. al., 2006). Y es que el tipo de evaluación utilizado por el profesorado de EF llega a depender incluso de la etapa educativa donde ejerzan. Según Sicilia, Sáenz-López, Manzano y Delgado (2009), mientras que el profesorado de educación secundaria prioriza la evaluación de aprendizajes conceptuales y procedimentales, las y los docentes de educación primaria otorgan mayor importancia a la evaluación del aprendizaje actitudinal. Además, según estos autores, las evaluaciones de uno u otro tipo implican el uso de diferentes medios e instrumentos que permitan realizar valoraciones adaptadas a dichos contenidos.

Sin embargo, más allá de los estudios anteriores, son escasas las investigaciones empíricas sobre la evaluación en EF escolar (López-Pastor, 2000b). Parece necesario, pues, ahondar en las experiencias del alumnado de FIPEF. Las fuertes raíces con que están asentadas muchas de las creencias del alumnado de Magisterio exigen entrar a considerar y analizar las experiencias de este alumnado porque pueden condicionar y reproducir enfoques y tipos de evaluación en EF cuando sean profesores y profesoras en activo (LópezPastor et al., 2004). Por tanto, atendiendo a estas circunstancias, el objetivo principal de este trabajo consiste en conocer las experiencias de un grupo de estudiantes universitarios sobre la evaluación de la EF durante su escolarización.

\section{METODOLOGÍA}

\subsection{EL ESTUDIO Y LOS INSTRUMENTOS}

Este artículo se corresponde con un estudio cualitativo, realizado con los relatos biográficos de un grupo de estudiantes universitarios, que se ocupa de las experiencias vividas en los procesos de evaluación de sus años de escolarización previa. La idea consiste en explorar las semejanzas y diferencias en sus experiencias, porque de esta manera estamos en disposición de crear conocimiento sobre los temas centrales y comunes al grupo de informantes de este estudio. Al analizar conjuntamente diferentes relatos individuales, podemos (re)construir una historia de vida colectiva que nos permita comprender las características de la evaluación en la EF en un periodo histórico determinado.

Para acceder a las experiencias de los participantes empleamos dos instrumentos metodológicos: los relatos y los grupos de discusión. Los relatos son documentos 
producidos por una persona que narra alguna experiencia biográfica, tal y como las ha vivido (Massot, Dorio \& Sabariego, 2004). Por grupo de discusión entendemos aquella "técnica no directiva que tiene por finalidad la producción controlada de un discurso por parte de un grupo de sujetos que son reunidos, durante un espacio de tiempo limitado, a fin de debatir sobre determinado tópico propuesto por el investigador" (Gil, 1992, pp. 200-201). Con la combinación de ambas técnicas podemos alcanzar un conocimiento más profundo que nos permita contrastar y enriquecer la información (Massot et al., 2004).

El estudio se sustenta con supuestos onto-epistemológicos relativistas porque, de acuerdo con otros autores (Pérez-Samaniego et al., 2011), consideramos que existen múltiples realidades socialmente construidas y, por tanto, accedemos a un conocimiento falible y subjetivo de dichas realidades. De esta manera, los resultados obtenidos no definen tanto la realidad objetiva de lo que ocurrió con la evaluación de los años de escolarización de los participantes, sino la realidad subjetiva que comparten estos estudiantes y que deriva de sus percepciones y recuerdos. Además, la información que proporcionan los participantes no la entendemos como un producto generado exclusivamente por las personas que la cuentan, sino como una suerte de construcción social del colectivo de estudiantes, pero que lo expresan individualmente con sus propias palabras. Desde este punto de vista, los relatos sobre las experiencias de los estudiantes son personales y, a la vez, sociales, porque, como señala Bolívar (2014), se incrustan en un contexto cultural determinado del que toman los recursos, ideas y experiencias compartidas para dar forma concreta a dichos relatos.

\subsection{PARTICIPANTES}

La muestra del estudio se compuso íntegramente por estudiantes de Didáctica de la Educación Física en la Educación Primaria (asignatura troncal de $2^{\circ}$ curso del grado en Maestro/a en Educación Primaria), impartida en la Facultad de Magisterio de la Universidad de Valencia durante el curso 2014-2015. Se empleó un muestreo de carácter no probabilístico intencional seleccionándose uno de los 11 grupos de dicha asignatura dada la facilidad de acceso. Del total de 48 estudiantes matriculados en la asignatura, 45 formaron parte de la muestra del estudio ( 36 alumnas y 9 alumnos ${ }^{1}$ ). Sus edades oscilaron entre los 19 años y los 25 años, siendo 19 y 20 las edades más frecuentes (40 estudiantes).

$\mathrm{Al}$ encontrarnos en la situación de que el investigador principal del estudio era también docente del alumnado participante, se tuvo especial atención en remarcar la voluntariedad de la participación en las actividades propuestas para la investigación y que, por lo tanto, en ningún caso la calificación de la asignatura iba a verse afectada por la decisión personal y particular de participar o no ni por los contenidos que en ellas se manifestaran. Asimismo, se garantizó la utilización ética de los datos obtenidos, la privacidad y confidencialidad de las personas participantes.

\subsection{PROCEDIMIENTO DE RECOGIDA DE DATOS}

Durante las últimas semanas del mes de septiembre de 2014, coincidiendo con el inicio del curso académico, se le solicitó al alumnado participante que redactara un relato en el que describiera los procesos de evaluación vividos en EF durante su educación obligatoria.

La dispar proporción de género se debe a que en los estudios de magisterio hay una mayor presencia femenina. 
Para garantizar la reflexividad de los mismos se les dio una serie de orientaciones: que distinguieran las etapas de primaria, secundaria y bachillerato, que abordaran las finalidades de la evaluación, así como los momentos en que se producían, las personas implicadas, los instrumentos y procesos. El alumnado dispuso de una semana para elaborar sus relatos.

Los grupos de discusión se realizaron durante la última semana de noviembre. De acuerdo con las orientaciones de Gil (1992), se establecieron ocho grupos de discusión, de entre 5 y 6 personas cada uno. El mayor inconveniente que encontramos lo determinó el hecho de que la muestra formaba parte de un mismo grupo de estudiantes, por lo que se conocían entre sí. Tratamos de compensar esa limitación facilitando un clima de seguridad y confianza. Para ello, se permitió la libre agrupación del alumnado manteniendo los cupos de componentes arriba descritos. Las sesiones tuvieron una duración aproximada de 2 horas y tuvieron lugar en el seminario de Didáctica de la Expresión Corporal de la Facultad de Magisterio. El profesor de la asignatura actuó de moderador gestionando los contenidos de la discusión y asignando los turnos de intervención. Todas las discusiones fueron registradas con una grabadora Philips Pocket Memo DPM6700.

Por último, durante el mes de diciembre se formaron 7 nuevos grupos de discusión (denominados a partir de ahora reflexiones grupales ${ }^{2}$ para diferenciarlos de los anteriores). Cada nuevo grupo estuvo compuesto por todas las personas que ocuparon el mismo asiento en sus respectivos grupos de discusión. De esta manera se pretendió que en las reflexiones grupales hubiera representación de cada grupo de discusión. Estas nuevas formaciones se emplearon para reflexionar sobre los contenidos de una serie de extractos de los grupos de discusión seleccionados por el investigador principal. Estas sesiones tuvieron una duración aproximada de una hora y fueron nuevamente grabadas en audio.

\subsection{PROCESO DE ANÁLISIS}

Todas las grabaciones fueron transcritas a lo largo del mes de agosto, dicha labor la realizó el mismo investigador que dirigió las reuniones de grupo, hecho que contribuyó tanto a facilitar las tareas de análisis e interpretación de los discursos, como a reducir la pérdida de información que supone el paso de datos orales a escritos (Requena, 2014).

El proceso de análisis cualitativo de datos consiste en la imbricación e interacción de tres operaciones básicas, la reducción de información, la exposición de los datos y la verificación de conclusiones (Massot, Dorio \& Sabariego, 2004). Para las labores de selección, focalización y abstracción en unidades de significado nos servimos del programa de análisis cualitativo NVivo v.10. A la hora de codificar y categorizar se siguió un proceso mixto. Por un lado, se identificó de forma deductiva una serie de unidades de análisis relacionadas con los modelos de evaluación educativa. Sin embargo, en la medida en que nuestra intención no se limitaba a la mera verificación de teorías, durante el proceso de análisis de los discursos fueron emergiendo de forma inductiva nuevas categorías de análisis. Finalmente se seleccionaron aquellas categorías y subcategorías más relevantes para nuestro estudio (Tabla 1).

A la hora de mostrar las declaraciones de las y los participantes, y con el objetivo de identificarlas preservando su anonimato, se ha empleado una serie de códigos identificativos formados por una letra y un número. Junto a cada cita textual, aparece $\mathrm{GDn}^{\circ}-, \mathrm{RGn}^{\circ}$ - o RI- según se trate de información extraída de los grupos de discusión, reflexiones grupales o relatos individuales respectivamente. Tras el guion aparece el código asignado a la persona participante. De esta forma, el código GD1-A5 corresponde al grupo de discusión número uno, participante A5. 
Tabla 1. Categorías y subcategorías seleccionadas para el estudio

\begin{tabular}{|l|l|}
\hline CATEGORÍA & SUBCATEGORÍA \\
\hline Ámbito de evaluación & $\begin{array}{l}\text { Contenidos curriculares evaluados } \\
\text { Tipos de conocimientos evaluados } \\
\text { Porcentajes de evaluación/calificación }\end{array}$ \\
\hline Procedimientos de evaluación & $\begin{array}{l}\text { Medios de evaluación de conocimientos prácticos } \\
\text { Medios de evaluación de conocimientos teóricos } \\
\text { Medios de evaluación de conocimientos actitudinales }\end{array}$ \\
\hline Criterios de evaluación & - \\
\hline
\end{tabular}

No obstante, en la medida en que la fragmentación del discurso produce una pérdida de información esencial al obviar el poder de la situación y las estrategias culturales y simbólicas que envuelven a cada sujeto (Martín, 2014), se ha realizado una segunda lectura de las declaraciones aportadas por los sujetos participantes del estudio -esta vez en su conjunto-, para recoger posibles inconsistencias y ambivalencias, así como para detectar las fuerzas y tensiones culturales y contextuales que influyen en sus argumentos.

\section{RESULTADOS Y DISCUSIÓN}

A continuación, se presentan los resultados más significativos del estudio estructurados a partir de la agrupación de diferentes categorías y subcategorías.

\subsection{QUÉ SE EVALÚA: CONTENIDOS Y CONOCIMIENTOS EVALUADOS}

Los resultados de este estudio muestran que la evaluación de la EF que vivieron los estudiantes universitarios en FIPEF se ocupaba especialmente de unos contenidos y marginaba a otros. Así, la evaluación se dedicaba a los contenidos curriculares considerados importantes (juegos, deportes, condición física) y, en menor medida, a otros contenidos (expresión corporal, las actividades en el medio natural, etc.). Esta diferenciación ya se observó en estudios previos sobre el desarrollo curricular en la EF escolar (López-Pastor, 1999; Molina \& Devís, 2001; Sicilia et al., 2009).

En particular, las experiencias de los informantes muestran que la evaluación se centraba en contenidos diferentes según la etapa educativa de primaria o secundaria a la que se referían. Mientras que en la etapa de primaria la evaluación recaía básicamente en contenidos como los juegos y las habilidades motrices básicas, en la educación secundaria se centraba mayoritariamente en la mejora de la condición física y las habilidades deportivas. En menor medida, se mencionan contenidos expresivos, así como otros contenidos relacionados con las actividades en el medio natural y la salud:

lo que pasa a todos, que [...] en primaria lo que se trata de evaluar más que nada es que si participas [en los juegos] y si los niños adquieren unas destrezas motoras, más 
que nada porque hacen juegos de correr, de saltar y demás. No evalúan pruebas físicas, porque no tienen capacidad para eso. En la Educación Secundaria Obligatoria (ESO), por ejemplo, sí que se evalúan las pruebas físicas que te hacen, por ejemplo tanto de correr como de saltos, lanzamiento de peso [...] flexibilidad y todo eso (RG2-B1).

En secundaria al final del [primer] trimestre [se evaluaba la] carrera continua, en el segundo trimestre el examen fue de resistencia, haciendo abdominales, flexiones y un ejercicio de ponerte a cuatro patas y levantar la pierna. [...] en primaria la forma de evaluar era el juego en sí, si lo hacías mejor o peor (GD5-G5).

Autores como Arbogast (2002), Dejong, Kokinakis y Kuntzleman (2002) y Hay (2006) encuentran fuertes conexiones entre la definición y concepción de la EF escolar, es decir los saberes que constituyen la asignatura, y su evaluación. De tal forma, parece lógico que los contenidos en los que más se centra la evaluación en cada una de las etapas correspondan a aquellos tradicionalmente dominantes, así puede observarse en los currículos oficiales de las últimas reformas en el sistema educativo (Molina \& Devís, 2001; Viciana \& Mayorga, 2013; Viciana, Salinas \& Cocca, 2007).

La gran relevancia de la evaluación para nuestro estudio es que pone en evidencia con claridad lo que el profesorado de EF considera importante enseñar y aprender en primaria y secundaria, y eso resulta difícil de comprender sin entender que responden a dos culturas profesionales distintas (Moreno, 2002). El profesorado de cada etapa cuenta con formas de entender, interactuar y trabajar diferentes porque entiende desigualmente su misión profesional. Cada grupo de profesionales pertenece a una comunidad epistémica distinta que promueve valores, prácticas y enseñanzas poco concordantes. Por un lado, la EF en la educación primaria suele tener un enfoque lúdico y recreativo a través de la cual se desarrollan habilidades y destrezas motrices, así como relaciones socio-afectivas. Mientras que, por otro lado, en la etapa de secundaria la EF se orienta hacia un contenido deportivo y de condición física (Sicilia et al., 2009).

Pero más allá de los contenidos curriculares (juegos, deportes, condición física, etc.), la evaluación también difiere según se trate de los conocimientos prácticos, teóricos o actitudinales de la asignatura. Por lo general, los aspectos teóricos suelen tener poca presencia en la evaluación de la EF escolar y parecen corresponder exclusivamente a la etapa de secundaria, tal y como se observa en estas citas:

[...] en primaria y en secundaria sí que noté diferencias a la hora de evaluar, porque en primaria sí que se tenía en cuenta la práctica y nada la teoría, y en secundaria, sobre todo en los últimos cursos, ya comenzaba a tenerse más en cuenta la teoría (GD2-A2).

sí que veo que en la ESO ya se le da más peso a la teoría de la EF, más que nada [...] para poderla equiparar con otras asignaturas (RG1-A2).

Igualmente, es destacable que prácticamente la única situación en la que la evaluación de los conocimientos teóricos cobraba verdadera relevancia se producía en los casos en los que el alumnado, por motivo de enfermedad o lesión, no podía participar en los procesos de evaluación convencionales. Así lo recoge esta cita: "En cuanto a los alumnos que no podían participar en las clases prácticas debido a enfermedades, esguinces... su nota final 
dependía del examen teórico y de un trabajo especial que tenían que hacer sobre las clases prácticas" (RI-B2).

Por lo que respecta a la evaluación de la EF en educación secundaria, algunos participantes de nuestro estudio señalan la menor relevancia de los conocimientos teóricos respecto a los prácticos. En algunos casos, incluso mencionan el peso porcentual asignado a cada tipo de conocimiento, como indican en estas citas:

[...] en secundaria sí que es verdad que empezamos con los libros de teoría y tal y teníamos un examen, pero sinceramente, ese examen tenía muy bajo porcentaje para la nota global (GD2-D2).

La parte práctica es lo que más contaba, creo que era un 60\% [...] después estaba el examen teórico, que era un $30 \%$ y después si te duchabas y todo eso era un $10 \%$ más de la nota (GD2-E2).

Estudios anteriores ya mostraron la poca relevancia del componente teórico en la evaluación de la EF escolar, así como las diferencias entre primaria y secundaria (Sicilia et al., 2006). En dichos estudios se indica que el aumento de la exigencia cognoscitiva al pasar de una etapa a la otra vendría determinado por la mayor orientación academicista que tiene la EF en la educación secundaria.

Por último, aunque los contenidos conceptuales podían estar presentes en la evaluación de EF, los participantes señalan la desconexión existente entre la teoría y la práctica vista en la asignatura:

[...] el examen era sobre eso, sobre el libro de texto, no sobre lo que tú hacías en clase práctica, sino preguntas del libro de texto (GD5-E5).

[...] el examen [...] no tenía ninguna relación, porque es que encima fue sobre deportes: baloncesto, balonmano... y no tenían relación [con la práctica] porque es que justo ese año no dimos ningún deporte. No sé si es que quiso complementar un poco esa parte de ese modo, no dio explicación, nos dio los contenidos en un día [...] nos lo tuvimos que empollar e hicimos el examen (GD1-G1).

Esta separación entre el conocimiento teórico y el práctico es un problema que arrastra la asignatura debido a que históricamente se ha construido sobre la experiencia práctica y poca reflexión teórica que la sustente (Devís-Devís, Martínez \& Villamón, 2002; Lawson, 1984). Posteriormente, este problema de desconexión entre el conocimiento teórico y el práctico se ha puesto de manifiesto en la EF escolar desde distintos lugares y momentos históricos diferentes (Devís \& Peiró, 2004; Stolz \& Pill, 2016).

Frente a este modelo inconexo, Velázquez y Hernández (2004) destacan la importancia de emplear procesos de evaluación que permitan conocer la funcionalidad y aplicación práctica de los conocimientos teóricos. Autores como Hay (2006) y López y Moreno (2002) abogan por una evaluación integrada, basada los procesos cognitivos y psicomotrices presentes en las distintas manifestaciones de la actividad física, superando así el dualismo que tradicionalmente envuelve nuestra asignatura.

En lo que a la evaluación de los conocimientos actitudinales se refiere, también encontramos diferencias entre las etapas de primaria y secundaria. Por lo general la actitud está mucho más valorada en la etapa de primaria, tal como se refleja a continuación: 
En primaria [...] es lo que más se tiene en cuenta [...] los valores que se quieren inculcar a los niños: [...] que sepa comportarse, que sepa respetar a los compañeros, la cuestión de la integridad, socialización. Pero ya en cuanto llegas a secundaria y bachiller, [...] ese aspecto actitudinal se pierde un poco en lo que es la evaluación (RG7-F5).

En resumen, la evaluación de EF en primaria miraba más por las actitudes que por las aptitudes [motrices]. [...] La EF en los cursos de ESO y $1^{\circ}$ de Bachillerato cambió radicalmente. La asignatura empezó a evaluarse justo al contrario que en la etapa anterior. La aptitud ganaba peso frente a la actitud (RI-F1).

Estos resultados están en sintonía con los hallados por Sicilia et al. (2006) que pusieron de manifiesto cómo el profesorado de primaria le otorga más relevancia que el de secundaria a la evaluación de contenidos actitudinales en la EF. Este hecho podría deberse a una mayor necesidad por parte del profesorado de primaria de controlar la conducta del alumnado y de transmitir determinados hábitos de orden e higiene, mientras que en secundaria la preocupación profesional se dirige a los resultados académicos. Autores como Viciana y Mayorga (2013) argumentan esta diferencia de planteamiento en que el alumnado de secundaria comienza a desarrollar más su condición física y habilidades deportivas, por lo tanto la asignatura está más centrada en la transmisión (y evaluación) de conocimientos práctico-procedimentales y no tanto en la de otros de corte más social y actitudinal.

\subsection{CÓMO SE EVALÚA: MEDIOS DE EVALUACIÓN}

En el apartado anterior se aprecia la tendencia existente en la EF por parcelar los aprendizajes, según estos respondan a conocimientos teóricos, prácticos o actitudinales. Esta tendencia también acaba por afectar a la evaluación, ya que cada uno de los conocimientos anteriores suelen evaluarse de forma independiente, con unos medios de evaluación diferenciados.

Los medios de evaluación son definidos como las producciones del alumnado (en tanto que sujeto evaluado) que el profesorado (en su función de evaluador) recoge y registra, y que sirven para apreciar el aprendizaje adquirido en un proceso educativo determinado (Hamodi, López-Pastor \& López-Pastor, 2015; Pérez-Pueyo et al., 2017).

\subsubsection{Los medios de evaluación empleados para valorar los conocimientos prácticos}

Los medios de evaluación empleados para valorar los conocimientos prácticos difirieron según se tratara de la etapa de primaria o secundaria (Tabla 2). 
Tabla 2. Medios prácticos de evaluación

\begin{tabular}{|l|l|l|}
\hline & \multicolumn{1}{|c|}{$\begin{array}{c}\text { Medios de evaluación más } \\
\text { utilizados }\end{array}$} & $\begin{array}{c}\text { Medios de evaluación menos } \\
\text { utilizados }\end{array}$ \\
\hline Educación primaria & $\begin{array}{l}\text { Demostraciones prácticas: } \\
\text { i) juegos motrices } \\
\text { ii) habilidades motrices básicas } \\
\text { Exámenes prácticos (a partir de } \\
5^{\circ} \text { de primaria) }\end{array}$ & Exámenes prácticos (hasta $5^{\circ}$ ) \\
\hline $\begin{array}{l}\text { Educación secundaria } \\
\text { y bachillerato }\end{array}$ & $\begin{array}{l}\text { Exámenes prácticos: } \\
\text { i) Pruebas de condición física } \\
\text { ii) Pruebas deportivas analíticas } \\
\text { iii) Representaciones expresivas }\end{array}$ & $\begin{array}{l}\text { Exámenes prácticos: } \\
\text { i) Pruebas deportivas globales }\end{array}$ \\
\hline
\end{tabular}

Así, durante la etapa de primaria, la evaluación del ámbito motriz consistía fundamentalmente en demostraciones prácticas (normalmente en forma de juegos) que el profesorado observaba de forma directa durante las clases prácticas. En alguna ocasión el alumnado mencionó haber realizado exámenes prácticos, aunque estos solían reservarse para los últimos cursos $\left(5^{\circ}\right.$ y $6^{\circ}$ de primaria):

En estos cursos predominaban los juegos, como por ejemplo torito en alto, balón prisionero, el pañuelo, bancos equilibristas, bote-bote, con zancos y a lo loco, la cadena, que no caiga el balón, etc. En cambio, en $5^{\circ}$ y $6^{\circ}$ de Primaria, al principio de cada trimestre se realizaban unas pruebas físicas de elasticidad, resistencia, salto, fuerza, equilibrio y velocidad (RI-C6).

[...] en primaria me acuerdo que pruebas físicas la única que hice fue correr. No sé si nos hicieron correr 30 o 35 minutos, [...] fue de los últimos cursos, no sé si sería $5^{\circ}$ o $6^{\circ}$. Y luego, durante el resto de curso, de $1^{\circ}$ a $6^{\circ}$ era lo que hacías en clase. $O$ sea, el juego que te hacía cada sesión (GD1-A1).

Por el contrario, en la etapa de educación secundaria se producía una mayor diversificación de medios práctico-procedimentales de evaluación. Por un lado, nuestros informantes destacaban las pruebas y test de capacidad física, empleados para evaluar la condición física del alumnado:

[...] la nota de cada trimestre salía de las pruebas, porque ella [la profesora] nos hacía las pruebas de velocidad, resistencia, balón medicinal, flexibilidad... Ella nos las hacía cada trimestre como para hacer un seguimiento (GD5-F5).

[...] teníamos unas pequeñas tablas súper grandes en que estaban todas las pruebas físicas y las actividades del curso, entonces las marcas las evaluaban con... cada marca correspondía a una nota ¿vale? Entonces estaba desde primero de la ESO hasta $1^{\circ}$ de bachillerato, entonces, tú siempre sabías las notas que tenías en todas las pruebas físicas (GD1-E1). 
En algunos casos estas pruebas fueron tan notables que condicionaron la forma de ver y entender la asignatura. Hasta el punto de identificarse con la asignatura como decía un participante: "esta asignatura solo tenía un nombre 'las pruebas físicas"” (RI-C3).

Según autores como Barbero (1996) y López-Pastor (2000a), estos medios de evaluación han predominado en las escuelas desde mitad del siglo pasado debido, entre otros factores, al peso de la costumbre y la tradición, la sencillez de su aplicación y su traducción en una nota y el halo de cientificidad y objetividad que los envuelve. A pesar de ello, autores como Keating (2003) y López-Pastor (2006) se muestran críticos con el uso de las pruebas de condición física como medio de evaluación en la EF escolar. Entre los argumentos esgrimidos destaca la exigua concordancia entre la información que proporcionan los test y los objetivos educativos de la EF, la escasa o nula información que aportan sobre los ámbitos cognitivo y emocional del alumnado, así como la poca conexión con la salud y estilos de vida activos. Asimismo, destacan el tedio y desmotivación que provocan entre el alumnado, la inexistencia de un consenso sobre la validez y veracidad de la información que proporcionan, el hecho de que su aplicación en clases numerosas requiera mucho tiempo, que generalmente se le resta a situaciones con mayor valor educativo. A pesar de que estas críticas han ido calando entre el profesorado de EF, Martínez, Zagalaz y Linares (2003) y López-Pastor (2013) mostraron que las pruebas de aptitud física siguen erigiéndose como medios necesarios para evaluar la condición física del alumnado.

Otro medio de evaluación destacado por el alumnado fueron las pruebas de habilidad deportiva. Estas pruebas normalmente consistían en la ejecución de elementos técnicos deportivos de forma analítica y descontextualizada. Como comentaban en un grupo de discusión: "no se evaluaba cómo jugabas al baloncesto, sino que a lo mejor tenías que hacer cinco botes así, o tienes que meter tres veces canasta, no se evaluaba jugar al baloncesto, y así con todos los deportes" (GD3-C3).

Solamente algunas personas recuerdan haber sido evaluadas a través de situaciones contextualizadas de juego. En estos casos, normalmente se valoraba la aplicación de las distintas técnicas deportivas en un partido, como se indica en esta cita:

[...] si estábamos trabajando baloncesto, ellos lo que evaluaban era a través de un partido, el que tú eras capaz de llevar a cabo [...] lo que anteriormente te han explicado: el pase, el bote, el tiro. $O$ sea, que tengas una mecánica correcta de todos los pasos (GD6-A6).

Brown y Hopper (2006) denuncian que el profesorado con demasiada frecuencia evalúa la competencia deportiva a través de pruebas técnicas analíticas. Según dichos autores, este tipo de pruebas puede ser efectivo para valorar destrezas deportivas aisladas, pero no otros ámbitos presentes en el juego como el esfuerzo personal o el compromiso cognitivo. Una evaluación auténtica e integrada, a partir de la práctica de juegos deportivos, se revela como la manera más significativa para valorar los conocimientos adquiridos por el alumnado en materia deportiva, permitiendo comprobar in situ no solamente la ejecución técnica, sino también la comprensión de las situaciones de juego, así como la capacidad de toma de decisiones (Griffin, Mitchell \& Oslin, 1997; Oslin, Mitchell \& Griffin, 1998).

El caso de los contenidos expresivos y artísticos seguía, por lo general, una evaluación más global y contextualizada, normalmente en forma de composición coreográfica y mediante la observación: 
[...] nosotras, por ejemplo, [...] practicábamos distintos saltos de cuerda y al final, pues teníamos que hacer una coreografía donde estuvieran todos esos saltos, entonces evaluaban si los saltos los hacíamos bien y la imaginación a la hora de hacer la coreografía (GD3-G3).

[...] el tercer trimestre fue un baile, o podíamos elegir una coreografía, un teatro, un musical y mi grupo por ejemplo, hizo un musical de películas Disney (GD1-F1).

Este hecho parece coincidir con los datos aportados por Montávez (2011), quien halló que el profesorado de secundaria mayoritariamente evaluaba los contenidos expresivos a partir de la observación sistemática de manifestaciones expresivas. Podemos apreciar cómo las pruebas analíticas, supuestamente objetivas y neutras que se emplearon de forma hegemónica para evaluar los contenidos deportivos y de condición física, no resultan efectivas para evaluar las manifestaciones artístico-expresivas, puesto que estas representan componentes complejos del movimiento como la estética, la armonía o la creatividad, aspectos difícilmente medibles y objetivables. Sin embargo, estos contenidos también se evalúan con una observación sistemática que consiste en cuantificar categorías o aspectos desmembrados de una actividad global que permita aportar una calificación global cuantitativa.

Podemos concluir que generalmente los medios práctico-procedimentales que se emplearon para evaluar los últimos cursos de la educación primaria, la ESO y el bachillerato están en sintonía con algunas propuestas que tratan de mostrar ejemplos supuestamente estandarizados, sistematizados y validados, dependiendo de si se trata de evaluar el desarrollo motor (Baena, Granero \& Ruiz, 2010), las habilidades motrices y coordinativas (Contreras, 1998; López \& Garoz, 2004), la condición y aptitud física (Castejón \& Alonso, 2004; Contreras, 1998; Pérez, 1994) o la destreza deportiva (Chinchilla \& Zagalaz, 1997). Este tipo de procedimientos normalmente están centrados en el resultado y suponen un modelo de evaluación inauténtico y descontextualizado (Hay, 2006), ineficaz para valorar las características más ricas y valiosas de la motricidad humana (López-Pastor, 2000a) y tendente a beneficiar a una élite motriz minoritaria y menoscabar al alumnado que no alcanza dichos patrones motrices (López-Pastor, 2006). Por tales motivos, en el ámbito escolar "la valoración e interpretación de los resultados de este tipo de pruebas [...] ha de relativizarse en función de las capacidades de cada alumno y alumna, de su trabajo y esfuerzo, y de las mejoras alcanzadas a lo largo el proceso" (Velázquez \& Hernández, 2004, p. 40).

\subsubsection{Los medios de evaluación empleados para valorar los aprendizajes teóricos}

Los medios de evaluación más utilizados para evaluar los contenidos teóricos (Figura 1) resultaron, en su mayoría, exámenes escritos, normalmente exámenes de tipo test o de respuesta breve:

[...] nos daban un cuadro, por ejemplo: Completa las dimensiones del campo de fútbol, por ejemplo, o: ¿Cuáles son las posiciones del voleibol? ¿Cada cuánto rotan?, o sea, preguntas también de desarrollar, de escribir y también de tipo test [...] el reglamento, la duración de un partido, por ejemplo, o cuáles son los objetivos del baloncesto, por ejemplo, o cuáles son las reglas del baloncesto [...] técnicas no tanto, porque [...] creo que era más [...] a la hora de la práctica [...] tácticas... poco también (GD2-B2). 
Figura 1. Medios teóricos de evaluación

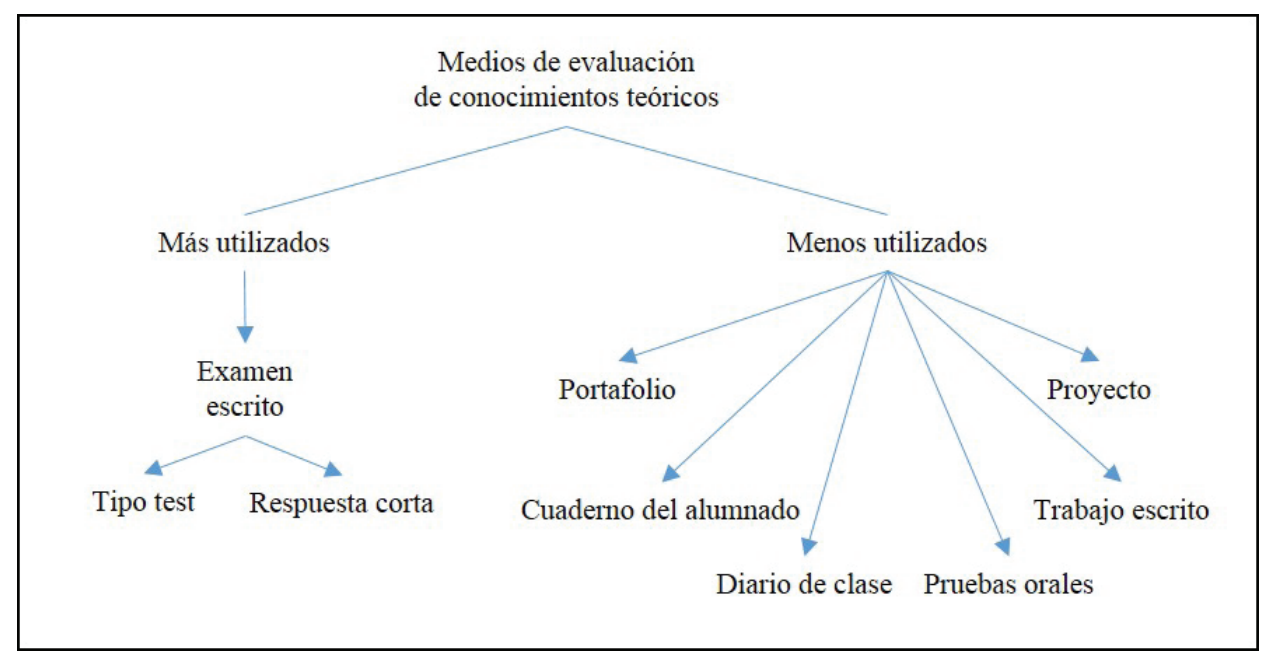

En algún caso se optó por preguntas de comprensión, aunque las más comunes eran memorísticas, normalmente referidas a cuestiones sobre aspectos deportivos, fisiológicos y anatómicos:

[...] teníamos un examen teórico de, por ejemplo, 4 temas [...] por ejemplo eran los deportes que habíamos tratado: fútbol, vóley y baloncesto. Y, por ejemplo, en el examen te preguntaban las dimensiones del campo, los objetivos del deporte... y, entonces, eso te lo tenías que memorizar del libro (GB2-B2).

[...] te ponían dibujos de tipos de estiramiento y tenías que indicar cuál era el músculo que se estaba estirando y cómo se llamaba el músculo. Y luego tenías que dibujar tú otro tipo de estiramiento que no... que no apareciera en el examen (GD3-A3).

En raras ocasiones se emplearon medios como cuadernos del alumnado, diarios de clase o trabajos escritos. En cualquier caso, estos fueron algo más recurrentes en la etapa de secundaria, y estaban dirigidos a profundizar sobre los temas que se estaban desarrollando en clase: "[en el instituto] a lo mejor, cuando estábamos tratando el tema del voleibol, pues nos hacía investigar sobre si era un deporte que se jugaba mucho en España, si era deporte olímpico, si... cosas así. Y sí, a lo mejor era un trabajo de 3 páginas y éramos grupos de 3 o 4 [...] pero no era un trabajo muy denso, ni..." (GD4-D4).

En un único caso se le solicitó al alumnado que redactara un portafolio. Como comenta la estudiante, en el portafolio debía recopilar tanto las actividades del curso, como las evidencias de su aprendizaje: "en bachiller [...] tuvimos que hacer un portafolios con... de cada actividad lo que habías aprendido" (GD3-E3). De igual manera, fueron escasas las ocasiones en las que el alumnado debió realizar proyectos de aprendizaje tutorado. En este sentido, una estudiante señala cómo tuvo que diseñar un plan de mejora de la condición física: 
[...] recuerdo que realizamos un trabajo en el que debíamos diseñar un entrenamiento, que debía durar 7 semanas, en el que planeábamos una serie de ejercicios teniendo en cuenta la teoría, es decir, las cualidades físicas básicas, de acuerdo a nuestras capacidades y, a la vez, progresivo con tal de mejorarlas. Además, toda esta teoría era luego aplicada a la práctica que conllevaba, por tanto, pruebas físicas (RI-B3).

Tampoco parecen frecuentes los medios de evaluación orales, estos se limitaron a preguntas en clase y exposiciones a lo largo del curso:

[...] hubo una temporada que nos tuvimos que saber todos los huesos y todos los músculos del cuerpo. Entonces en ese caso, a lo mejor, antes de comenzar la clase, cuando estábamos haciendo lo del calentamiento [...] nos decía: Recuerda a tus compañeros cuáles eran los músculos y qué estás estirando en cada caso. O [...] si estábamos dando algo relacionado: ¿Cómo se llamaba este saque? (GD2-A2).

Nuestros resultados difieren de los presentados por Sicilia et al. (2006) quienes encontraron que los trabajos escritos, seguidos del cuaderno del alumnado, representan los principales medios de evaluación de conceptos teóricos en EF tanto en la etapa de primaria como de secundaria, mientras que los exámenes de tipo test fueron los menos utilizados, si bien es cierto que estos fueron más frecuentes en primaria que en secundaria.

Gran parte de los resultados hallados en relación con la evaluación de la dimensión conceptual contravienen las recomendaciones de López-Pastor (2000a) y López y Moreno (2002). Estos autores proponen una evaluación más orientada a la comprensión que a la memorización, empleando para ello instrumentos interactivos como el diálogo, discusiones, resolución de planteamientos problemáticos, etc.

\subsubsection{Los medios de evaluación empleados para valorar los aprendizajes actitudinales}

Por último, los procesos de evaluación del ámbito actitudinal (Figura 2) destacan por su uniformidad. Como podemos apreciar, tanto en primaria como en secundaria solían estar mediatizados por la observación de la conducta del alumnado durante las clases de EF y la adquisición de hábitos de higiene como el aseo personal. Por el contrario, ninguna alumna o alumno manifestó que se hubieran empleado medios como entrevistas personales, asambleas o actividades de role-playing:

En primaria [...] suelen evaluar, por ejemplo, si llevas la bolsa de aseo a clase, si te portas bien en clase, si has participado (RG6-A6).

[en secundaria] lo de la actitud [se evaluaba] sobre todo, cuando jugábamos en equipo. [...] si la profesora veía que había alguna pelea o algo sí que lo tenía en cuenta, pero... más que nada [durante] la práctica (GD3-G3).

Estos resultados están en sintonía con los mostrados en Sicilia et al. (2006) en tanto que la observación in situ durante las actividades de aprendizaje fue una práctica habitual para la evaluación de las actitudes. Asimismo, el profesorado participante en su estudio manifestó que el cuaderno del alumnado no resultó un medio habitual de evaluación. 
Figura 2. Medios de evaluación del ámbito actitudinal

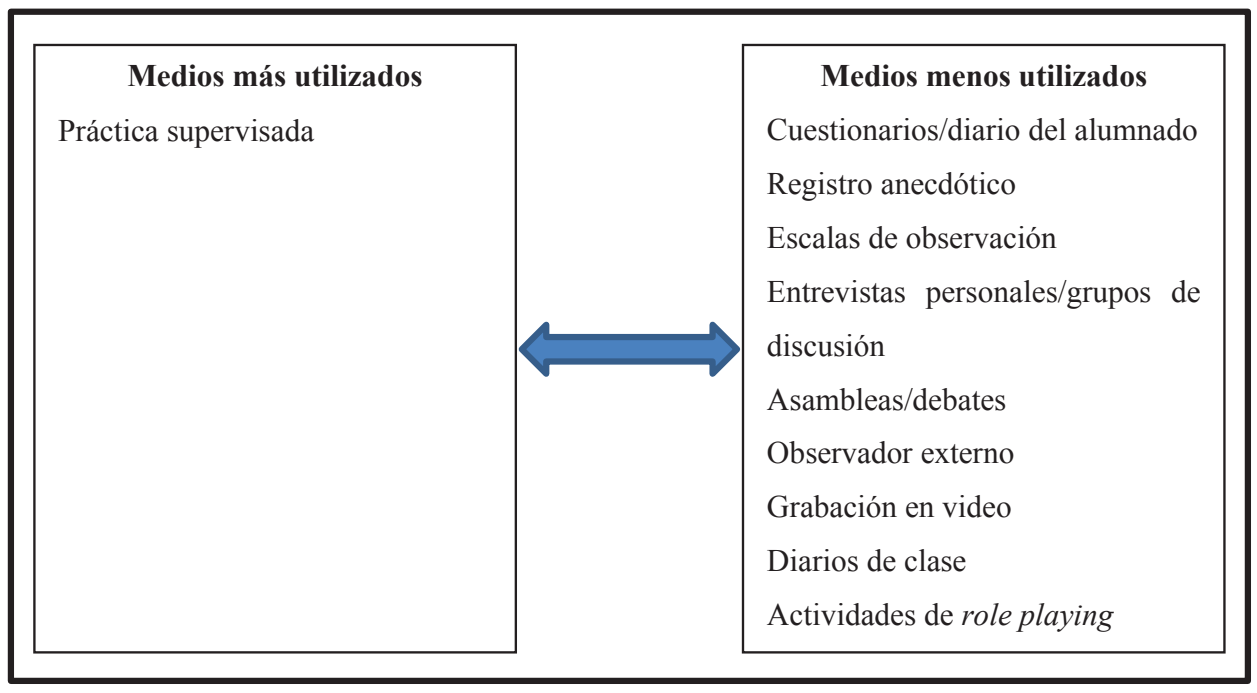

La escasez de medios de evaluación que el alumnado experimentó en la evaluación del plano actitudinal en EF contrasta con las líneas marcadas por Velázquez y Maldonado (2004). Estos autores defienden que la evaluación de dicho ámbito tiene un carácter problemático, ya que se trata de una dimensión de la conducta humana difícilmente observable, que puede fluctuar a lo largo del tiempo.

\subsection{LOS CRITERIOS DE EVALUACIÓN: TIPOS DE APRENDIZAJE}

Los criterios de evaluación, es decir, los aspectos que se toman como referencia para emitir juicios de valor sobre el aprendizaje del alumnado, vienen determinados por los conocimientos considerados deseables que deben adquirirse en su proceso educativo. Estos criterios, aunque se indican en los currículos oficiales, pasan por la interpretación y el tamiz 'ideológico' del profesorado que es, en última estancia, quien termina de concretarlos en sus programaciones y aplicarlos en las distintas tareas evaluativas.

A tenor de nuestros datos, normalmente el profesorado no informaba al alumnado de los criterios de evaluación que iba a seguir a lo largo de los procesos educativos o la información proporcionada estaba incompleta. Estos son dos ejemplos que apoyan esta idea:

[...] muchas veces no sabíamos qué estaba evaluando, qué era lo que buscaba con ese ejercicio o qué notas nos había puesto hasta el final del trimestre con la entrega de las notas (RP-B5).

[...] en los deportes y en acrogimnasia, sí que te decía qué iba a valorar, en plan: el esfuerzo, la originalidad, que fuera algo así... que no fuera un churro, vaya. Pero lo que no sabías concretamente de dónde había salido la nota (GD1-G1). 
A pesar de que el alumnado no pudo informarnos acerca de los criterios de evaluación empleados por su profesorado, es posible deducirlos si tenemos en cuenta, por un lado, qué consideraba importante evaluar y, por el otro, cómo lo hacía. Por tanto, no es de extrañar que las diferencias encontradas anteriormente entre las etapas de primaria y secundaria se vean reflejadas en los criterios de evaluación.

Por un lado, la evaluación de la EF en primaria (sobre todo, los cuatro primeros cursos) solía articularse mediante criterios orientados a la participación activa en las actividades y juegos propuestos: "[en la evaluación durante la etapa de primaria] los profesores le daban más importancia a que entre todos jueguen, o sea, cooperen, colaboren entre todos, que no haya discriminación entre unos y otros" (RG3-C2).

Estos datos son coherentes con el enfoque lúdico y vivencial por los que se caracterizó la LOGSE 3 (Viciana \& Mayorga, 2013). Según estos autores, la LOGSE potenció una EF (y, por tanto, una evaluación) basada en las vivencias, la recreación y la participación del alumnado en las clases. Este enfoque nace como reacción a la anterior ley educativa (LGE) caracterizada por estar centrada en la consecución de una serie de logros de aprendizaje motor y de condición física, y que predisponían a una evaluación final, sumativa y normativa.

Por el contrario, la tendencia en la etapa de secundaria fue emplear criterios de evaluación orientados a medir el rendimiento motor a partir de la mejora de la condición física o las habilidades deportivas. Así se observa en este comentario: "[en secundaria] se evaluaba [...] lo que eras, o sea, si tú eras muy capaz en la EF, pues sacabas buena nota, y eso era valorado y si no lo eras, no" (RG7-G1).

Encontramos cierta sintonía entre nuestros resultados y los criterios de evaluación propuestos por el currículum oficial de secundaria y bachillerato. Así, por ejemplo, entre los criterios planteados para $3^{\circ}$ de la ESO se encuentra: "Incrementar los niveles de resistencia aeróbica, flexibilidad y fuerza resistencia a partir del nivel inicial, al participar en la selección de las actividades y ejercicios en función de los métodos de entrenamiento propios de cada capacidad" (p. 30491). También resulta llamativo el siguiente criterio de bachillerato: "Haber perfeccionado las habilidades específicas correspondientes a un deporte, mostrando eficacia, técnica y táctica, en la resolución de los problemas que plantea su práctica" (p. 71341). Más adelante el currículum especifica: "Con este criterio se pretende comprobar si los alumnos han alcanzado un nivel de habilidad motriz específica que les permita desenvolverse con autonomía y con cierta eficacia en un determinado deporte, de entre los que se practican en su entorno, que responda a sus características particulares y motivaciones personales" (p. 71341).

Este tipo de criterios basados en el rendimiento, lejos de ser inocuos para el alumnado, tienden a beneficiar a un grupo de estudiantes -al que podríamos considerar como la élite motriz-, y deja por el camino a otro tipo de personas menos competentes que, a fuerza de obtener bajos resultados, acaban adquiriendo un sentimiento de incapacidad abocándolos a la objeción (Barbero, 1996).

Gran parte de las y los participantes manifestaron que para evaluar estos criterios centrados en el rendimiento motor, se utilizaban diferentes baremos en función del género:

Las leyes educativas vigentes mientras los participantes del estudio cursaban la etapa de primaria fueron la LOGSE (desarrollada en la Comunidad Valenciana por el decreto 20/1002) y la LOCE (que nunca llegó a concretarse en un currículum oficial). Cuando pasaron a la educación secundaria se produjo un nuevo cambio de ley, aprobándose la LOE y desarrollándose en el marco territorial de la Comunidad Valenciana dos currículos independientes, uno para la Educación Secundaria Obligatoria (decreto 112/2007) y otro para el bachillerato (decreto 102/2008). 
"cuando eran pruebas de resistencia, la velocidad, flexibilidad, ahí sí que nos decían: Tenéis que hacer esto para conseguir tal nota, chicas por una parte, chicos por otra y luego os evaluaré diferenciados" (GD5-A5). De acuerdo nuevamente con Barbero (1996) esta práctica contribuye a apuntalar las diferencias en los roles de género e institucionaliza la inferioridad femenina.

\section{CONCLUSIONES}

Una vez analizados los resultados, podemos afirmar que las experiencias de evaluación de EF escolar vividas por las personas participantes solían tener ciertos rasgos comunes. Entre ellos, destaca que el profesorado tendía a evaluar de forma diferenciada los conocimientos prácticos, teóricos y actitudinales, utilizando, para ello, medios diferenciados. En la ecuación «Evaluación = Práctica + Teoría + Actitud», el término práctico solía guardar un marcado grado de prelación sobre los otros dos. Esta tendencia puede revertirse en favor de aspectos actitudinales en la etapa de primaria.

La evaluación de los conocimientos prácticos se caracterizó por estar mayoritariamente centrada en los contenidos hegemónicos para cada una de las etapas educativas: juegos y habilidades motrices básicas en la educación primaria, y condición física y deportes en la etapa secundaria. Igualmente se encontraron diferencias en los medios de evaluación más utilizados en cada una de las etapas. Mientras que en primaria predominó la participación en las propias actividades de aprendizaje, en secundaria fueron más comunes los test de actitud física y las pruebas de técnicas deportivas.

La evaluación de los conocimientos teóricos tuvo mayor relevancia en la educación secundaria que en la primaria, en la que prácticamente no se produjo. Los medios de evaluación más utilizados fueron los exámenes escritos, bien de tipo test, bien de respuesta breve, y normalmente estaban referidos a cuestiones anatómicas, fisiológicas y deportivas.

La evaluación de los conocimientos actitudinales tuvo más presencia en la etapa de primaria que en la de secundaria. Los contenidos evaluados solían ser la actitud del alumnado durante las actividades y el cumplimiento de una serie de normas higiénicas. Como medios de evaluación, el profesorado solía valerse de las propias tareas de aprendizaje.

Por último, comprobamos que los sistemas de evaluación que el profesorado empleaba en sus clases guardaban coherencia con los criterios de evaluación formulados por los documentos oficiales vigentes en su momento, que establecían los currículos de la EF en la Comunidad Valenciana.

A tenor de los resultados obtenidos, podemos observar que generalmente las experiencias de evaluación de EF durante las etapas de educación primaria y secundaria que vivió el alumnado de FIPEF, participante en nuestro estudio, se enmarcan en un paradigma tradicional. Es decir, una evaluación que corresponde a una concepción técnica del currículum, tanto en su diseño como en su desarrollo (Álvarez, 2001; López-Pastor, 2000a).

Los criterios de credibilidad y rigor del estudio vinieron determinados por la triangulación metodológica de los datos, pues se recogió información de diferentes sujetos, a través de distintas técnicas en varios momentos. Ello nos permitió confrontar la información recabada, detectar ambivalencias y consistencias en el discurso. Entre las principales limitaciones del estudio podemos destacar que para ajustarnos a los 
requerimientos de publicación, nos hemos visto obligados a seleccionar un número reducido de categorías de análisis, relegando otras que igualmente emergieron durante el análisis a ulteriores publicaciones. Por lo tanto, resulta complejo hacerse una idea global y detallada de las experiencias de evaluación vividas por los participantes. Está en nuestro ánimo continuar esta línea de investigación para ofrecer una visión más amplia de los sistemas de evaluación en EF vividos por las y los participantes.

Este estudio puede resultar interesante para el personal docente y/o investigador con interés en la FIPEF, concretamente por la influencia de las experiencias vividas en la etapa de estudiante y su posible transferencia en la práctica profesional. También puede servir como un punto de partida para planificar la enseñanza de la didáctica de la EF para el alumnado de FIPEF, en cuanto a la evaluación se refiere, estableciendo conexiones entre las experiencias vividas, la adquisición de conocimientos y competencias profesionales y la cultura profesional. Con ello, esperamos iniciar un proceso reflexivo que sirva para orientar el proceso de enseñanza-aprendizaje.

Como futuras líneas de investigación, consideramos importante abordar las experiencias vividas sobre evaluación en la EF y su influencia en la práctica docente una vez las y los egresados de FIPEF ejerzan profesionalmente, y así comprobar el grado en que las facultades de educación contribuyen a la reproducción de propuestas de evaluación tradicionales o bien a construir modelos alternativos.

\section{REFERENCIAS BIBLIOGRÁFICAS}

Álvarez Méndez, J. M. (2001). Evaluar para conocer, calificar para excluir. Madrid: Morata.

Arbogast, G. A. (2002). Assessment issues and the elementary school-age child, Part I. Journal of Physical Education, Recreation \& Dance, 73(7), 21-25.

Baena, A., Granero, A. \& Ruiz, P. J. (2010). Procedimientos e instrumentos para la medición y evaluación del desarrollo motor en el sistema educativo. Journal of Sport and Health Research, 2(2), 63-75.

Barbero, J. I. (1996). Cultura profesional y currículum (oculto) en educación física. Reflexionar sobre las (im)posibilidades del cambio. Revista de Educación, 311, 13-49.

Bolívar, A. (2014). Las historias de vida del profesorado: voces y contexto. Movimento, 19(62), 711734.

Brown, S., \& Hopper, T. (2006). Can all students in Physical Education get an 'A'? Game performance assessment by peers as a critical component of student learning. Physical and Health Education, 72(1), 14-21.

Castejón, F. J., \& Alonso, D. (2004). Evaluación de las capacidades físicas básicas. En J. L. Hernández Álvarez y R. Velázquez Buendía (Coords.), La evaluación en educación física. Investigación y práctica en el ámbito escolar (pp. 77-106). Barcelona: Graó.

Chinchilla, J. L., \& Zagalaz, M. L. (2007). Educación física y su didáctica en primaria. Torredonjimeno (Jaén): Jabalcuz.

Conselleria de Educación. (1992). Decreto 20/1992, de 17 de julio, del Gobierno valenciano, por el que se establece el currículum de la Educación Primaria en la Comunidad Valenciana. DOGV, 1728.

Conselleria de Educación. (2007). Decreto 112/2007, de 20 de julio, del Consell, por el que se establece el currículo de la Educación Secundaria Obligatoria en la Comunidad Valenciana. DOGV, 5562, 30402-30587.

Conselleria de Educación. (2008). Decreto 102/2008, de 11 de julio, del Consell, por el que se 
establece el currículum del Bachillerato en la Comunidad Valenciana. DOGV, 5806, 7130371341.

Contreras, O. R. (1998). La evaluación de la educación física en la educación primaria. En O. R. Contreras, Didáctica de la Educación física. Un enfoque constructivista (pp. 293-312). Barcelona: Inde.

Dejong, G., Kokinakis, C. L., \& Kuntzleman, C. (2002). The role of assessment in meeting the NASPE physical education content standards. Journal of Physical Education, Recreation \& Dance, 73(7), 22-25.

Devís-Devís, J., Martínez-Moya, P., \& Villamón, M. (2002). La profesionalización de la educación física española: caracterización y evolución del conocimiento científico. En S. García Blanco (Coord.), Congreso Internacional de Historia de la Educación Física (pp. 149-156). Madrid: Gymnos.

Devís-Devís, J., \& Peiró-Velert, C. (2004). Los materiales curriculares en la educación física. En A. Fraile (Coord.), Didáctica de la educación física. Una perspectiva crítica y transversal (pp. 6394). Madrid: Biblioteca Nueva.

Doolittle, S. A., Dodds, P., \& Placek, J. H. (1993). Persistence of beliefs about teaching during formal training of preservice teachers. Journal of teaching in Physical Education, 12(4), 355-365.

Gil, J. (1992). La metodología de investigación mediante grupos de discusión. Enseñanza \& Teaching. Revista Interuniversitaria de Didáctica, 10-11, 199-212.

Gómez, V., \& Guerra, P. (2012). Teorías implícitas respecto a la enseñanza y el aprendizaje: ¿Existen diferencias entre profesores en ejercicio y estudiantes de pedagogía? Estudios Pedagógicos, 38(1), 25-43.

Griffin, L. L., Mitchell, S. A., \& Oslin, J. L. (1997). Teaching sport concepts and skills: A tactical games approach. Champaign, IL: Human Kinetics.

Hamodi, C., López-Pastor, V. M., \& López-Pastor, A. T. (2015). Medios, técnicas e instrumentos de evaluación formativa y compartida del aprendizaje en educación superior. Perfiles educativos, 37(147), 146-161.

Hay, P. J. (2006). Assessment for learning in physical education. En D. Kirk, D. Macdonald y M. O'Sullivan (Eds.), The Handbook of Physical Education (pp. 312-325). Londres: Sage.

Johnson, D. (2010). Identidad y formación docente de los profesores principiantes. Un enfoque biográfico-narrativo (tesis de maestría). Universidad de Chile, Santiago de Chile.

Keating, X. D. (2003). The current often implemented fitness test in Physical Education programs: problems and future directions. Quest, 55, 141-160.

Lawson, H. A. (1984). Invitation to Physical Education. Champaign: Human Kinetics.

López Estévez, R. (2014). Paradigmas y fundamentos de la evaluación en educación física: retrospectiva y prospectiva. E-motion. Revista de Educación, Motricidad e Investigación, 2, 53 77.

López-Pastor, V. M. (1999). Prácticas de evaluación en educación física: estudio de casos en primaria, secundaria y formación del profesorado. Valladolid: Universidad de Valladolid.

López-Pastor, V. M. (2000a). Buscando una evaluación formativa en educación física: Análisis crítico de la realidad existente, presentación de una propuesta y análisis general de su puesta en práctica. Apunts. Educación Física y Deportes, 62, 16-26.

López-Pastor, V. M. (2000b). La evaluación en la Educación Física en España: una revisión bibliográfica (1970-1997). Habilidad motriz. Revista de Ciencias de la Actividad Física y el Deporte, 16, 4-14.

López-Pastor, V. M. (2006). La evaluación en educación física. Revisión de los modelos tradicionales y planteamiento de una alternativa: la evaluación formativa y compartida. Buenos Aires: Miño y Dávila.

López-Pastor, V. M. (2013). Nuevas perspectivas sobre evaluación en educación física. Revista de educación física, 29(3), 1-10. 
López-Pastor, V. M., García-Peñuela, A., Pérez Brunicardi, D., López-Pastor, E., \& Monjas Aguado, R. (2004). Las historias de vida en la formación inicial del profesorado de Educación Física. Revista Internacional de Medicina y Ciencias de la Actividad Física y el Deporte, 4(13), 45-57.

López-Pastor, V. M., Kirk, D., Lorente-Catalán, E., MacPhail, A., \& Macdonald, D. (2013). Alternative assessment in physical education: a review of international literature. Sport, Education and Society, 18(1), 57-76.

López, C., \& Garoz, I. (2004). Evaluación de las capacidades coordinativas. En J. L. Hernández Álvarez y R. Velázquez Buendía (Coords.), La evaluación en educación física. Investigación y práctica en el ámbito escolar (pp. 107-135). Barcelona: Graó.

López, A., \& Moreno, J. A. (2002). Aprendizaje de hechos y conceptos en educación física. Una propuesta metodológica. Apunts. Educación Física y Deportes, 69, 18-26.

Martín, E. (2014). Mentiras, inconsistencias y ambivalencias. Teoría de la acción y análisis del discurso. Revista Internacional de Sociología, 72(1), 115-138. doi:10.3989/ris.2012.07.24

Martínez, L. (1994). Influencia de las creencias implícitas en la formación inicial de los especialistas en Educación Física. En S. Romero (Coord.), Didáctica de la Educación Física: diseños curriculares en primaria: ponencias y comunicaciones del I Congreso Nacional de Educación Física de Facultades de Ciencias de la Educación y XII de Escuelas U. de Magisterio (pp. 225232). Sevilla: Wanceulen Editorial Deportiva.

Martínez, E. J., Zagalaz, M. L., \& Linares, D. (2003). Las pruebas de aptitud física en la evaluación de la Educación física de la ESO. Apunts. Educación Física y Deportes, 71, 61-77.

Massot, I., Dorio, I., \& Sabariego, M. (2004). Estrategias de recogida y análisis de la información. En R. Bisquerra (Coord.), Metodología de la investigación cualitativa (pp. 329-366). Madrid: La Muralla.

Molina, J. P., \& Devís-Devís, J. (2001). La educación física en la reforma educativa actual: análisis crítico. En B. Vázquez (Coord.), Bases educativas de la actividad física y el deporte (pp. 301321). Madrid: Síntesis.

Montávez, M. (2011). La expresión corporal en la realidad educativa. Descripción y análisis de su enseñanza como punto de referencia para la mejora de la calidad docente en los centros públicos de Educación Primaria de la ciudad de Córdoba (Tesis doctoral no publicada). Universidad de Córdoba, Córdoba.

Moreno, T. (2002). Cultura profesional del docente y evaluación del alumnado. Perfiles educativos, 24(96), 23-36.

Oslin, J. L., Mitchell, S. A., \& Griffin, L. L. (1998). The game performance assessment instrument (GPAI): development and preliminary validation. Journal of Teaching in Physical Education, 17(2), 231-243.

Pérez, J. P. (1994). Autoevaluación de las capacidades físicas. Aula, 19 (suplemento Aula 23), 3-18.

Pérez-Pueyo, Á., López-Pastor, V. M., Hortigüela, D., \& Gutiérrez, C. (2017). Aclaración de los términos implicados en el proceso de evaluación educativa. En V. M. López-Pastor y Á. PérezPueyo (Coords.), Evaluación formativa y compartida en educación: experiencias de éxito en todas las etapas educativas (pp. 70-91). León: Universidad de León.

Pérez-Samaniego, V. M., Devís-Devís, J., Smith, B. M., \& Sparkes, A. (2011). La investigación narrativa en la educación física y el deporte: qué es y para qué sirve. Movimento, 17(1), 11-38.

Prieto, A. (2015). Los paradigmas de evaluación en Educación Física. Multiárea. Revista de didáctica, 7, 110-130.

Requena, M. (2014). La transcripció, una escolta que es fa text i un text que escolta. Arxius, 31, 107-124.

Santos, M. Á. (2000). La escuela que aprende. Madrid: Ediciones Morata.

Sicilia, A., Delgado, M. A., Sáez-López, P., Manzano, J. I., Varela, R., Cañadas, J. F., \& Gutiérrez, M. (2006). La evaluación de aprendizajes en Educación Física. Diferencias en función del nivel educativo. Motricidad. European Journal of Human Movement, 17, 71-95. 
Sicilia, A., Sáenz-López, P., Manzano, J. I., \& Delgado, M. A. (2009). El desarrollo curricular de la Educación Física en primaria y secundaria: un análisis desde la perspectiva del profesorado. Apunts. Educación Física y Deportes, 98, 23-32.

Stolz, S.A., \& Pill, S. (2016). Telling Physical Education Teacher Education Tales Through Pedagogical Case Studies. Sport, Education and Society, 21(6), 868-887.

Tillema, H. H. (2000). Belief change towards self-directed learning in student teachers: Immersion in practice or reflection on action. Teaching and Teacher Education, 16(5), 575-591.

Velázquez, R., \& Hernández, J. L. (2004). Evaluación en educación y evaluación del aprendizaje en educación física. En J. L. Hernández Álvarez y R. Velázquez Buendía (Coords.), La evaluación en educación física. Investigación y práctica en el ámbito escolar (pp. 11-47). Barcelona: Graó.

Velázquez, R., \& Maldonado, A. (2004). Las actitudes y su evaluación en educación física. En J. L. Hernández y R. Velázquez (coord.), La evaluación en educación física. Investigación y práctica en el ámbito escolar (pp. 196-203). Barcelona: Graó.

Viciana, J., \& Mayorga-Vega, D. (2013). Análisis del cambio curricular de la LOGSE a la LOE en la educación física de primaria. Profesorado. Revista de currículum y formación del profesorado, 17(3), 257-271.

Viciana, J., Salinas, F., \& Cocca, A. (2007). Análisis de contenido comparativo del primer nivel curricular de Educación Física en Secundaria. Profesorado. Revista de currículum y formación del profesorado, 11(2), 1-13. 
